\title{
Softly massive gravity
}

\author{
G. Gabadadze \\ Center for Cosmology and Particle Physics, Department of Physics, New York University, New York, New York 10003, USA* \\ and Theory Division, CERN, CH-1211 Geneva 23, Switzerland \\ M. Shifman \\ Theory Division, CERN, CH-1211 Geneva 23, Switzerland \\ and William I. Fine Theoretical Physics Institute, University of Minnesota, Minneapolis, Minnesota 55455, USA ${ }^{\dagger}$
}

(Received 30 December 2003; published 29 June 2004)

\begin{abstract}
Large-distance modification of gravity may be the best mechanism for solving the cosmological constant problem. A simple model of the large-distance modification-four-dimensional (4D) gravity with a hard mass term-is problematic from the theoretical standpoint. Here we discuss a different model, brane-induced gravity, that effectively introduces a soft graviton mass. We study the issues of unitarity, analyticity, and causality in this model in more than five dimensions. We show that a consistent prescription for the poles of the Green's function can be specified so that 4D unitarity is preserved. However, in certain instances 4D analyticity cannot be maintained when the theory becomes higher dimensional. As a result, one has to sacrifice 4D causality at distances of the order of the present-day Hubble scale. This is a welcome feature for solving the cosmological constant problem, as was recently argued in the literature. We also show that, unlike 4D massive gravity, the model has no strong-coupling problem at intermediate scales.
\end{abstract}

DOI: $10.1103 /$ PhysRevD.69.124032

PACS number(s): 04.50.+h

\section{LARGE-DISTANCE MODIFICATION OF GRAVITY: FORMULATING THE PROBLEM}

The reason underlying the observed acceleration of the Universe is puzzling. It could be a tiny amount of vacuum energy. However, this possibility is hard to reconcile with known particle-physics models. Instead, it might well be that a new physical scale exists in the gravitational sector and the laws of gravity and cosmology are modified at this scale. To be consistent with data and be able to predict the accelerated expansion, the new scale should be roughly equal to $H_{0}^{-1}$ $\sim 10^{28} \mathrm{~cm}$ - the present-day value of the Hubble length. In this regard, developing models in which gravity gets modified at cosmological distances becomes a timely endeavor. A generally covariant theory of the large-distance modification of gravity is the Dvali-Gabadadze-Porrati (DGP) model [1]. The gravity action of the model can be written as follows:

$$
S=\frac{M_{\mathrm{Pl}}^{2}}{2} \int d^{4} x \sqrt{g} R(g)+\frac{M_{*}^{2+N}}{2} \int d^{4} x d^{N} y \sqrt{\bar{g}} \mathcal{R}_{4+N}(\bar{g}),
$$

where $R$ and $\mathcal{R}_{4+N}$ are the four-dimensional and $(4+N)$-dimensional Ricci scalars, respectively, and $M_{*}$ stands for the gravitational scale of the bulk theory. Extra dimensions are not compactified; they asymptote at infinity to Minkowski space. The higher-dimensional and fourdimensional metric tensors are related as

$$
\bar{g}(x, y=0) \equiv g(x) \text {. }
$$

\footnotetext{
*Present address.

${ }^{\dagger}$ Permanent address.
}

The first term on the right-hand side of Eq. (1) acts as a kinetic term for a 4D graviton while the second term acts as a gauge invariant mass term. The observable matter is assumed to be localized on a 4D surface $y=0$.

The present work is devoted to the study of the DGP scenario in the case $N \geqslant 2$ (see Refs. [2,3]). Such models have a string theory realization [4]. More importantly, these models are potential candidates for solving $[5,6]$ the cosmological constant problem (see also Refs. [7-24] for interesting cosmological and astrophysical studies).

The equation of motion for the theory described by the action (1) takes the form

$$
\delta^{(N)}(y) M_{\mathrm{Pl}}^{2} G_{\mu \nu}^{(4)} \delta_{A}^{\mu} \delta_{B}^{\nu}+M_{*}^{2+N} G_{A B}^{(D)}=-T_{\mu \nu}(x) \delta_{A}^{\mu} \delta_{B}^{\nu} \delta^{(N)}(y) .
$$

Our conventions are as follows:

$$
\begin{aligned}
& \eta_{A B}=\operatorname{diag}[+--\cdots-], \quad A, B=0,1, \ldots, 3+N, \\
& \mu, \nu=0,1,2,3, \quad a, b=4,5, \ldots, 3+N .
\end{aligned}
$$

$G_{\mu \nu}^{(4)}$ and $G_{A B}^{(D)}$ denote the four-dimensional and $D$-dimensional Einstein tensors, respectively. We choose (for simplicity) a source localized on the brane, $T_{\mu \nu}(x) \delta^{(N)}(y)$.

Gravitational dynamics encoded in Eq. (3) can be inferred from both the four-dimensional (4D) as well as $(4+N)$-dimensional standpoints. From the 4D perspective, gravity on the brane is mediated by an infinite number of Kaluza-Klein $(\mathrm{KK})$ modes that have no mass gap. Under conventional circumstances (i.e., with no brane kinetic term) this would lead to higher-dimensional interactions. However, the large 4D Einstein-Hilbert $(\mathrm{EH})$ term suppresses the wave functions of heavier KK modes, so that in effect they do not 
participate in the gravitational interactions on the brane at observable distances [25]. Only light KK modes, with masses $m_{\mathrm{KK}} \lesssim m_{c}$,

$$
m_{c} \equiv \frac{M_{*}^{2}}{M_{\mathrm{Pl}}},
$$

remain essential, and they collectively act as an effective 4D graviton with a typical mass of the order of $m_{c}$ and a certain smaller width.

Assuming that $M_{*} \sim 10^{-3} \mathrm{eV}$ or so, we obtain $m_{c} \sim H_{0}$ $\sim 10^{-42} \mathrm{GeV}$. Therefore, the DGP model with $N \geqslant 2$ predicts [26] a modification of gravity at short distances $M_{*}^{-1}$ $\sim 0.1 \mathrm{~mm}$ and at large distances $m_{c}^{-1} \sim H_{0}^{-1} \sim 10^{28} \mathrm{~cm}$, give or take an order of magnitude. Since gravitational interactions, nevertheless, are mediated by an infinite number of states at arbitrarily low energy scale, the effective theory (1) presents, from the 4D standpoint, a nonlocal theory [5]. Moreover, as was suggested in [27], nonlocalities postulated in pure $4 D$ theory can solve an "old" cosmological constant problem [27], and give rise to new mechanisms for the present-day acceleration of the Universe [27,28]. (It is interesting to note that the nonlocalities in a gravitational theory that are needed to solve the cosmological constant problem could appear from quantum gravity [29] or matter loops [30] in a purely $4 \mathrm{D}$ context.)

On the other hand, from the $(4+N)$-dimensional perspective, gravitational interactions are mediated by a single higher-dimensional graviton. This graviton has two kinetic terms given in Eq. (1), and, therefore, can propagate differently on and off the brane; namely, at short distances, i.e., at $r<m_{c}^{-1} \sim H_{0}^{-1} \sim 10^{28} \mathrm{~cm}$, the graviton emitted along the brane essentially propagates along the brane and mediates $4 \mathrm{D}$ interactions. However, at larger distances, the extradimensional effects take over and gravity becomes $4+N$ dimensional.

As was first argued in Ref. [2], the results in $N \geqslant 2$ DGP models are sensitive to ultraviolet (UV) physics, in contradistinction to the $N=1$ model [1]. In other words, one should either consistently smooth out the width of the brane [26], or introduce a manifest UV cutoff in the theory [26,31,32], or do both. With a finite thickness, more localized operators appear on the worldvolume of the brane, in addition to the worldvolume Einstein-Hilbert term already present in Eq. (1) [26]. For instance, one could think of a higher-dimensional Ricci scalar smoothly spread over the worldvolume [3].

In general, terms that are square of the extrinsic curvature can also emerge. Some of these terms can survive in the limit when the brane thickness tends to zero (i.e., in the lowenergy approximation). For instance, in the zero-thickness limit of the brane the following terms might be important:

$$
\begin{gathered}
\delta^{(N)}(y) h_{\mu}^{\mu} \partial_{\alpha}^{2} h_{a}^{a}, \quad \delta^{(N)}(y) h^{\mu \nu} \partial_{\mu} \partial_{\nu} h_{a}^{a}, \\
\delta^{(N)}(y) h_{\mu}^{\mu} \partial_{a} \partial_{b} h^{a b}
\end{gathered}
$$

where $h$ denotes small perturbations on flat space. Although the main features of the model, such as interpolation between the $4 \mathrm{D}$ power-law behavior of a nonrelativistic potential at short distances and the higher-dimensional behavior at large distances, are not expected to be changed by adding these terms, nevertheless, the tensorial structure of a propagator could in general depend on these terms and self-consistency of the theory may require some of these terms to be present in the actions in a reparametrization invariant way.

In the low-energy approximation the exact form of these "extra" terms and their coefficients are ambiguous, because of their UV origin. They will be fixed in a fundamental theory from which the DGP model can be derived [4,33]. In the present paper, in the absence of such a fundamental theory (but in the anticipation of its advent), we would like to study a particular parametrization of these "extra" terms, for demonstrational purposes. According to our expectations, physics in the self-consistent theory will have properties very similar to those discussed below. We will show that these properties are rather attractive since they do avoid severe problems of 4D massive gravity.

Consider the action

$$
\begin{aligned}
S= & \frac{M_{\mathrm{Pl}}^{2}}{2} \int d^{4} x \sqrt{g}\left[a R(g)+b \mathcal{R}_{4+N}\right] \\
& +\frac{M_{*}^{2+N}}{2} \int d^{4} x d^{N} y \sqrt{\bar{g}} \mathcal{R}_{4+N}(\bar{g}),
\end{aligned}
$$

where, in addition to the 4D EH term, a $D$-dimensional $\mathrm{EH}$ term localized on the brane is present. Here $a$ and $b$ are some numerical coefficients. We will study the properties of the system described by Eq. (7) for different values of $a$ and $b$. The action (7) is fully consistent with the philosophy of Ref. [1]: if there is a $(1+3)$-dimensional brane in $D$-dimensional space, with some "matter fields" confined to this brane, quantum loops of the confined matter will induce all possible structures consistent with the geometry of the problem, i.e., a $(1+3)$-dimensional wall embedded in $D$-dimensional space.

The equation of motion in the model (7) takes the form

$$
\begin{aligned}
& \delta^{(N)}(y) M_{\mathrm{Pl}}^{2}\left(a G_{\mu \nu}^{(4)}+b G_{\mu \nu}^{(D)}\right) \delta_{A}^{\mu} \delta_{B}^{\nu}+M_{*}^{2+N} G_{A B}^{(D)} \\
& \quad=-T_{\mu \nu}(x) \delta_{A}^{\mu} \delta_{B}^{\nu} \delta^{(N)}(y) .
\end{aligned}
$$

In deriving the above equation we first introduced a finite brane width $\Delta$, and then took the $\Delta \rightarrow 0$ limit in such a way that no surface terms appear. In general, the results depend on the regularization procedure for the brane width. In the present work, we adopt a simple prescription in which derivatives with respect to the transverse coordinates calculated on the brane vanish in the $\Delta \rightarrow 0$ limit (a unique prescription could be specified only by a fundamental theory). As previously, $G^{(4)}$ and $G^{(D)}$ denote the four-dimensional and $D$-dimensional Einstein tensors, respectively, while $a$ and $b$ are certain constants. In order to be able to describe 4D gravity at short distances with the right value of the Newton's coupling we set

$$
a+b=1 \text {. }
$$

Note that the first two terms in parentheses on the left-hand side of Eq. (8) can be identically rewritten as 


$$
\begin{array}{r}
(a+b) G_{\mu \nu}^{(4)}+b\left(-\partial_{\mu} \partial_{a} h_{\nu}^{a}-\partial_{\nu} \partial_{a} h_{\mu}^{a}-\partial_{a}^{2} h_{\mu \nu}+\eta_{\mu \nu} \partial_{a}^{2} h_{C}^{C}\right. \\
\left.+\partial_{\mu} \partial_{\nu} h_{a}^{a}-\eta_{\mu \nu} \partial_{\alpha}^{2} h_{a}^{a}+2 \eta_{\mu \nu} \partial_{a} \partial_{\alpha} h^{a \alpha}+\eta_{\mu \nu} \partial_{a} \partial_{b} h^{a b}\right) .
\end{array}
$$

The above equation of motion (8) — which should be viewed as a regularized version of the DGP model-could be obtained from the action (1) as well, provided the latter is amended by certain extrinsic curvature terms (for more details, see Ref. [33]). Below we will study this version of the regularized DGP model for various values of the parameters $a$ and $b$. Certain issues in the $a=0, b=1$ case, regularized with a finite brane width, have been recently analyzed in Ref. [3]. We will find below that phenomenologically more attractive is the $a=1, b=0$ case.

The issues to be addressed are as follows.

Assume that gravity measurements are done at points $x_{1}$ and $x_{2}$ that are confined to the brane. At distances $\left|x_{1}-x_{2}\right|$ $<H_{0}^{-1}$ the graviton propagator imitates that of a massive $4 \mathrm{D}$ unstable particle with mass $\sim m_{c}$. Given that the model itself is intrinsically $4+N$ dimensional, the following questions must be answered: (i) Does the graviton propagator $G\left(x_{1}, x_{2}, y=0\right)$ satisfy the requirements of four-dimensional unitarity? (ii) Do "abnormalities" occur at 4D momenta much smaller than the ultraviolet cutoff and larger than the infrared (IR) crossover scale, such as a precocious onset of the strong-coupling regime?

Needless to say, the answers to these questions determine whether the DGP model is intrinsically self-consistent and phenomenologically viable. The answer to the first question will be demonstrated to be positive and to the second negative. That is to say, the situation is most favorable. We hasten to add that it is not trivial to see that this is indeed the case. It is necessary to carry out a rather subtle analysis which circumvents stereotypes in, at least, one point.

For what follows it is instructive to confront the DGP model with 4D "hard" massive gravity [34] [or Pauli-Fierz (PF) gravity] which also leads to a large-distance modification of interactions. [The action of "hard" massive gravity is given below in Eq. (98).] In particular, we compare the perturbative treatments of these two models. Perturbation theory in Newton's constant in 4D massive gravity breaks down at a scale much lower than the cutoff scale of the theory-this was first obtained for spherically symmetric sources in Ref. [35]. The origin of this breaking can be traced back [36] to Feynman diagrams involving nonlinear interactions of gravitons. In terms of degrees of freedom, it is the longitudinal polarizations of the massive gravitons that are responsible for the perturbation theory breaking. This can be readily inferred from the dynamics of these modes analyzed in Ref. [37]. (Note also that the PF graviton propagates six degrees of freedom instead of five [38] in the full quantum theory. This makes the corresponding Hamiltonian unbound from below [38]. As a result, solutions exist that destabilize the empty Minkowski space [39] and the instability can develop practically instantaneously.)

As will be shown, in this respect the DGP gravity (7) presents a drastic improvement. In contradistinction with the 4D Pauli-Fierz theory of massive gravity, the precocious breakdown of perturbation theory does not occur in the DGP model with $N \geqslant 2$. A direct analogy with the Higgs mechanism for non-Abelian gauge fields is in order here.

For non-Abelian gauge fields with a hard gauge boson mass term, appropriate nonlinear amplitudes invalidate the perturbative expansion (i.e., violate the unitarity bound) at a scale set by the gauge boson mass divided by the gauge coupling constant. To cure this disaster, one introduces an extra scalar-the Higgs field. ${ }^{1}$ By the same token, certain nonlinear perturbative amplitudes of the 4D "hard" massive gravity blow up precociously [35-37] at a scale significantly lower than a naive UV cutoff of the theory under consideration. The unwanted growth of the amplitudes is canceled, however, in the DGP model at the expense of introducing an infinite number of the KK fields. Thus, the action (7) gives rise to a gravitational analogue of the Higgs mechanism, with an infinite number of "Higgs" fields which include both vector and scalar states.

It has been recently argued [40] that the spectrum of the DGP model contains tachyonic states with a negative norm ("tachyonic ghosts"). The conclusion was based on an analysis of the poles in the graviton propagator derived from the action (1). In fact, the analysis of Ref. [40] leaves aside subtle points of appropriately defining the Green's function poles. We formulate and discuss an appropriate rule for defining the would-be poles. With this rule accepted, the 4D unitarity of the Green's functions is guaranteed. This is not the case with respect to 4D analyticity and causality, though. In certain instances we will have to sacrifice 4D causality at distances of the order of today's Hubble scale. As was argued in Ref. [27] this is a welcome feature for solving the cosmological constant problem.

The organization of the paper is as follows. In Sec. II we discuss in detail a simplified version of the phenomenon, with a tensorial structure suppressed. We consider a scalar field with a Lagrangian similar to Eq. (1) and derive the Green's function. There are complex poles of the Green's function on the second and subsequent nonphysical Riemann sheets. This corresponds to the resonance nature of the 4D massive scalar. In Sec. III we discuss the same problem for gravity, i.e., including the graviton tensorial structure. The issue of proper definition of poles in the graviton propagator emerges in earnest in the trace part. In certain cases, a naive way of defining the poles leads to inconsistent resultsviolations of unitarity. For these cases we indicate a way out, treating the would-be poles in the Green's function in such a way that unitarity is not violated. Finally, in Sec. IV the $N$ $\geqslant 2$ DGP model is argued to have no strong-coupling problem at intermediate scales, in contradistinction to 4D PauliFierz gravity with a hard mass term.

\section{THE SIMPLEST EXAMPLE: SCALAR FIELD}

To warm up, we start our discussion with a simple model of a scalar field $\Phi$ in $(4+N)$-dimensional space-time. For

\footnotetext{
${ }^{1}$ The mass of the Higgs field itself needs a stabilization mechanism. This is a separate story, however.
} 
convenience, we separate the dependence of the scalar field $\Phi$ on four-dimensional and higher-dimensional coordinates $\Phi\left(x_{\mu}, y_{a}\right) \equiv \Phi(x, y)$. The two-kinetic-term action-the scalar counterpart of Eq. (1) - has the form

$$
\begin{aligned}
S= & \frac{M_{\mathrm{Pl}}^{2}}{2} \int d^{4} x \partial_{\mu} \Phi(x, 0) \partial^{\mu} \Phi(x, 0) \\
& +\frac{M_{*}^{2+N}}{2} \int d^{4} x d^{N} y \partial_{A} \Phi(x, y) \partial^{A} \Phi(x, y) .
\end{aligned}
$$

It is important to understand that in scalar case the analogue of the new term included in Eq. (8) but absent in Eq. (3) reduces, identically, to the already existing localized term. This is a consequence of our choice of the regularization of the brane width $\Delta$ and the boundary conditions according to which transverse derivatives vanish on the brane in the $\Delta$ $\rightarrow 0$ limit.

To study interactions mediated by the scalar field we assume that $\Phi$ couples to a source $J$ localized in the 4D subspace in a conventional way, $\int d^{4} x \Phi(x, 0) J(x)$. Then the equation of motion takes the form

$$
\delta^{(N)}(y) M_{\mathrm{Pl}}^{2} \partial_{\mu}^{2} \Phi(x, 0)+M_{*}^{2+N} \partial_{A}^{2} \Phi(x, y)=J(x) \delta^{(N)}(y) .
$$

The very same equation applies to the scalar field Green's function.

\section{A. Spectral representation}

First, using the scalar field example, we will summarize general arguments for the existence or absence of a spectral representation in higher-dimensional theories with the worldvolume kinetic terms as in Eq. (11). The explicit formulas below refer to the scalar case. In the next section we will consider gravity, with the appropriate tensorial structure, and will emphasize crucial differences between the present scalar example and full-blown gravity.

By the "spectral representation" we mean the KällenLehmann (KL) representation for the free tree-level propagator in the model (11) in terms of four-dimensional Mandelstam variables. Since the theory described by Eq. (11) is intrinsically higher dimensional, it is not clear a priori why the spectral representation in terms of the $4 \mathrm{D}$ variables should hold at all. Indeed, on the one hand, the KL representation expresses the fact that a given amplitude, as a function of $p^{2}$ (the 4D momentum squared), is analytic in the complex $p^{2}$ plane everywhere except for possible isolated poles plus a branch cut along the real positive semiaxis. On the other hand, the model (11) is nonlocal from the 4D standpoint; hence, it is not obvious why analyticity of the amplitude with respect to the $4 \mathrm{D}$ variable $p^{2}$ should occur in the regime where higher-dimensional effects become crucial.

There is an alternative point of view on the KL representation of the model (11). We can assume that the extra dimensions $y$ are compactified, with a finite (albeit arbitrarily large) compactification radius $R$. Then, the spectrum of the theory must consist of "discretized" Kaluza-Klein modes. From the 4D standpoint they are just certain massive states.
Then one could certainly obtain the KL representation by writing the (tree-level) 4D propagator summing up the entire tower of the KK eigenstates.

This strategy is readily implemented in the conventional compactifications, when the brane worldvolume term is absent. In this case the spectrum of the Kaluza-Klein eigenmodes

$$
\Phi(x, y)=\sum_{n} \Phi_{n}(x) \phi_{n}(y)
$$

becomes trivially discrete, with the eigenvalues $m_{n}^{2}$ $\sim n^{2} / R^{2}$. If so, the expression for the Green's function $G(p, y=0)$ takes the form

$$
G(p, y=0)=\sum_{n} \frac{\phi_{n}^{*}(0) \phi_{n}(0)}{m_{n}^{2}-p^{2}-i \epsilon}, \quad p^{2}=p_{\mu} p^{\mu} .
$$

In other words, $G(p, y=0)$ is the sum over an infinite number of poles, with positive-definite residues. As $R \rightarrow \infty$ the sum goes into the standard dispersion integral,

$$
G(p, y=0)=\frac{1}{\pi} \int_{0}^{\infty} d t \frac{\rho(t)}{t-p^{2}-i \epsilon},
$$

where $\rho(t)$ is a positive-definite spectral density.

The argument above, as well as the simple representation (14) or (15) following from it, neglects the existence of the brane worldvolume kinetic term in the action [the first term on the right-hand side of Eq. (11)]. This term is crucial and by no means can be neglected. It gives rise to kinetic mixings of the KK modes on the brane worldvolume,

$$
\frac{M_{\mathrm{Pl}}^{2}}{2} \int d^{4} x\left(\sum_{m} \partial_{\mu} \Phi_{m}(x) \phi_{m}(0)\right)\left(\sum_{n} \partial^{\mu} \Phi_{n}(x) \phi_{n}(0)\right) .
$$

Therefore, the KK modes defined in Eq. (13) are not the eigenstates of the Hamiltonian in the presence of the brane kinetic term. Diagonalization is needed. For the scalar field example (11), explicit diagonalization is possible and was in fact carried out [25]. As a result, the spectral representation can be argued to exist in the desired form Eq. (15).

In the case of gravity things are more complicated, however. The worldvolume $\mathrm{EH}$ term gives rise to kinetic mixings between the massive KK modes of distinct spins. It is not obvious how to diagonalize the full linearized Hamiltonian. Even if the diagonalization is possible, it is not clear whether the diagonal eigenstates are states of a definite 4D spin, and not the mixed states. This is all because of the large kinetic mixings between all the KK states on the brane. Thus, the spectral representation we look for is hard to obtain through explicit summation of the eigenstates of the Hamiltonian (if possible at all). The best one can do is to write down the spectral representations in the limiting regimes when the 4D EH term is either dominant or negligibly small. We will re- 
turn to this issue in Sec. III. Prior to delving into the gravity problem, we want to conduct detailed studies of the scalar example (11).

\section{B. Solving Eq. (12) in the general case}

To solve this equation it is convenient to Fouriertransform it with respect to "our" four space-time coordinates $x_{\mu} \rightarrow p_{\mu}$, keeping the extra $y$ coordinates intact. Marking the Fourier-transformed quantities by a tilde,

$$
\Phi(x, y) \rightarrow \widetilde{\Phi}(p, y),
$$

we then get from Eq. (12)

$$
\begin{aligned}
& \delta^{(N)}(y) M_{\mathrm{Pl}}^{2}\left(-p^{2}\right) \widetilde{\Phi}(p, 0)+M_{*}^{2+N}\left(-p^{2}-\Delta_{y}\right) \widetilde{\Phi}(p, y) \\
& \quad=\widetilde{J}(p) \delta^{(N)}(y),
\end{aligned}
$$

where $p^{2} \equiv p_{0}^{2}-p_{1}^{2}-p_{2}^{2}-p_{3}^{2}$, and the notation

$$
\Delta_{y} \equiv \sum_{a=1}^{N} \frac{\partial^{2}}{\partial y_{a}^{2}}
$$

is used. form:

We will look for the solution of Eq. (18) in the following

$$
\widetilde{\Phi}(p, y) \equiv D(p, y) \chi(p),
$$

where the function $D$ is defined as a solution of the equation

$$
\left(-p^{2}-\Delta_{y}-i \bar{\epsilon}\right) D(p, y)=\delta^{(N)}(y) .
$$

Note that the function $D$ is uniquely determined only after the $i \bar{\epsilon}$ prescription specified above is implemented. We also introduce the convenient abbreviation

$$
D_{0}(p) \equiv D(p, y=0) .
$$

Now, it is quite obvious that a formal solution of Eq. (18) can be written in terms of the function $D$ as follows:

$$
\widetilde{\Phi}(p, y)=-\frac{\widetilde{J}(p)}{M_{\mathrm{Pl}}^{2}} \frac{D(p, y)}{p^{2} D_{0}(p)-M_{*}^{2+N} / M_{\mathrm{Pl}}^{2}}+c \widetilde{\Phi}_{\mathrm{hom}}(p, y),
$$

where $\widetilde{\Phi}_{\text {hom }}(p, y)$ is a general solution of the corresponding homogeneous equation [i.e., Eq. (18) with a vanishing righthand side], and $c$ is an arbitrary constant. Equation (23) presents, in fact, the Green's function too, up to the factor $\widetilde{J}(p) / M_{\mathrm{Pl}}^{2}$, which must be amputated. In particular, for the Green's function on the brane we have

$$
\begin{gathered}
G(p, 0)=\frac{M_{\mathrm{Pl}}^{2}}{\widetilde{J}(p)} \widetilde{\Phi}(p, y=0), \\
G_{\mathrm{hom}}(p, 0)=\frac{M_{\mathrm{Pl}}^{2}}{\widetilde{J}(p)} \Phi_{\mathrm{hom}}(p, y=0),
\end{gathered}
$$

while for arbitrary values of $y$

$$
G(p, y)=-\frac{D(p, y)}{p^{2} D_{0}(p)-u^{N}}+c G_{\mathrm{hom}}(p, y),
$$

where

$$
u^{N} \equiv \frac{M_{*}^{2+N}}{M_{\mathrm{Pl}}^{2}}=m_{c}^{2} M_{*}^{N-2} .
$$

The presence or absence of the homogeneous part is regulated by the $i \epsilon$ prescription. Note that, if the first term on the right-hand side of Eq. (25) has poles on the real axis of $p^{2}$, then the homogeneous equation has a solution

$$
G_{\text {hom }}(p, y)=D(p, y) \delta\left(p^{2} D_{0}(p)-u^{N}\right) .
$$

This fact will play an essential role for gravity, as will be discussed in due course in Sec. III.

In what follows we will examine the poles of the Green's function $G(p, y)$. The positions of these poles depend on the functions $G_{\text {hom }}(p, y)$ and $D_{0}$ as defined in Eqs. (21) and (22). The choice of a particular rule of treatment of the poles corresponds to the choice of appropriate boundary conditions in the coordinate space. Note that the latter are dictated by physical constraints on the Green's function $G$ rather than on the auxiliary function $D$.

To get to the main point, we will try the simplest strategy of specifying the poles and check, a posteriori, whether this strategy is self-consistent. Let us put

$$
c=0
$$

and define $D$ in the Euclidean momentum space. Since in the Euclidean space the expression for $D$ is well defined and has no singularities,

$$
\begin{aligned}
D\left(p_{E}, q\right) & =\frac{1}{p_{E}^{2}+q^{2}}, \quad D\left(p_{E}, q\right) \equiv \int d^{N} y e^{i q y} D\left(p_{E}, y\right), \\
q^{2} & =\sum_{a}\left(q^{a}\right)^{2},
\end{aligned}
$$

one can perform analytic continuation from the Euclidean to the Minkowski space. This is not the end of the story, however. It is the Green's function $G$ that we are interested in, not the auxiliary function $D$. As will be explained below, the above procedure is consistent, for the following reason. The function $G$ obtained in this way has a cut extending from zero to infinity. In addition, we find two complex conjugate poles on the second nonphysical Riemann sheet of the complex $p^{2}$ plane. Moreover, there are additional poles on subsequent unphysical sheets.

Since the poles are not on the physical Riemann sheet, they do not correspond to any asymptotic states of the theory. A pole on the second Riemann sheet is a well-known signature of a resonance state [41]. Therefore, our toy scalar "gravity" is mediated by a massive resonance. The 
resonance-mediated gravity was first discussed in Refs. [42$44]$ in a different brane-world model.

Before passing to consideration of particular cases it is worth recalling that the Green's functions in the $N \geqslant 2$ DGP models need a UV regularization $[2,26,31,32]$. This has been mentioned already. An appropriate UV regularization can be achieved either by introducing an explicit UV cutoff, or, alternatively, by keeping a nonzero brane width in a consistent manner (defined in Ref. [26]). For brevity we choose the former prescription by consistently taking the limit of zero brane width. However, we should stress that all our results hold equally well in the brane-width regularization method of [26].

\section{Six dimensions}

It is instructive to demonstrate how things work by considering separately the six-dimensional case. In six dimensions, sensitivity to the UV cutoff is only logarithmic, and it is conceivable that the results obtained in the cutoff theory could be consistently matched to those of a more fundamental UV-completed theory to come. ${ }^{2}$

It is not difficult to calculate

$$
D_{0}(s)=\frac{1}{4 \pi} \ln \left(\frac{\Lambda^{2}}{-s}+1\right), \quad s \equiv p^{2},
$$

where $\Lambda^{2}$ is an ultraviolet cutoff. With this expression for $D_{0}$ the function $G\left(p^{2}, 0\right)$ develops a cut on the positive semiaxes of $s$ due to the logarithmic behavior of $D_{0}(s)$. This fact has a physical interpretation. Since the extra dimensions are noncompact in the model under consideration, the spectrum of the theory, as seen from the 4D standpoint, consists of an infinite gapless tower of the KK modes. This generates a cut in the Green's function for $s$ ranging from zero to $+\infty$.

In addition, there might exist isolated singular points in $G\left(p^{2}, 0\right)$. These singularities (for $s \ll \Lambda^{2}$ ) are determined by the equation

$$
G^{-1}(s, 0) \equiv s-m_{c}^{2}\left[\frac{1}{4 \pi} \ln \left(\frac{\Lambda^{2}}{-s}\right)\right]^{-1}=0,
$$

where $m_{c}^{2}$ is defined in Eq. (5). Let us introduce the notation

$$
s \equiv s_{0} \exp (i \gamma),
$$

where $s_{0}$ is a real positive number. Then, Eq. (30) has two solutions of the form

$$
s_{0} \approx 4 \pi m_{c}^{2}\left[\ln \frac{\Lambda^{2}}{m_{c}^{2}}\right]^{-1},
$$

and

\footnotetext{
${ }^{2}$ The $D>6$ models of brane-induced gravity are power sensitive to UV physics. In general one expects all sorts of higher-derivative operators in this case.
}

$$
\gamma_{1} \simeq-\frac{\pi}{\log \left(\Lambda^{2} / m_{c}^{2}\right)}, \quad \gamma_{2} \simeq 2 \pi+\frac{\pi}{\log \left(\Lambda^{2} / m_{c}^{2}\right)} .
$$

We conclude that there are two complex-conjugate poles on the nearby nonphysical Riemann sheets. These poles cannot be identified with any physical states of the theory. They are, in fact, manifestations of a massive resonance state. All other complex poles appear on subsequent nonphysical Riemann sheets.

\section{More than six dimensions}

Physics at $D>6$ is similar to that of the six-dimensional world which was described in Sec. II C. There are minor technical differences between odd- and even-dimensional spaces, however, as we will discuss momentarily.

In seven dimensions we find

$$
D_{0}(s)=\frac{1}{2 \pi^{2}}\left\{\Lambda-\sqrt{-s} \arctan -\left(\frac{\Lambda}{\sqrt{-s}}\right)\right\} .
$$

As in the 6D case, there is a branch cut. The cut in this case is due to the dependence of the Green's function on $\sqrt{s}$. No other singularities appear on the physical Riemann sheet. All poles are on nonphysical Riemann sheets, as previously.

In the eight-dimensional space the expression for $D_{0}$ reads

$$
D_{0}(s)=\frac{1}{16 \pi^{2}}\left\{\Lambda^{2}+s\left(\ln \frac{\Lambda^{2}}{-s}+1\right)\right\} .
$$

Again, we find a cut due to the logarithm, similar to that of the $6 \mathrm{D}$ case. All isolated singularities appear on nonphysical Riemann sheets.

The nine-dimensional formula runs parallel to that in seven dimensions,

$$
D_{0}(s)=\frac{1}{12 \pi^{3}}\left\{\frac{\Lambda^{3}}{3}+s\left(\Lambda-\sqrt{-s} \arctan \frac{\Lambda}{\sqrt{-s}}\right)\right\} .
$$

Finally, in ten dimensions

$$
D_{0}(s)=\frac{1}{128 \pi^{3}}\left\{\frac{\Lambda^{4}}{2}+s\left[\Lambda^{2}+s\left(\ln \frac{\Lambda^{2}}{-s}+1\right)\right]\right\} .
$$

The pole structure of $G$ is identical to that of the eightdimensional case. Since the pattern is now well established and clear-cut, there seems to be no need to dwell on higher dimensions.

Before turning to gravitons we would like to make comments concerning the UV cutoff $\Lambda$. The crossover distance $r_{c} \sim m_{c}^{-1}$ depends on this scale: in 6D the dependence is logarithmic, while in $D>6$ this dependence presents a power law $[2,4]$. Hence, the crossover scale in the $N \geqslant 2$ DGP models, unlike that in the $N=1$ model, is sensitive to particular details of the UV completion of the theory. Since in the present work we adopt an affective low-energy field-theory strategy, we are bound to follow the least favorable scenario 
in which the cutoff and the bulk gravity scale coincide with each other and both are equal to $M_{*} \sim 10^{-3} \mathrm{eV}$. If a particular UV completion were available, it could well happen that the UV cutoff and bulk gravity scale were different from the above estimate. In fact, in the string-theory-based construction of Ref. [4], the UV completion is such that the cutoff and bulk gravity scale are in the ballpark of $\mathrm{TeV}$.

In conclusion of this section it is worth noting that the Green's function $D_{0}$ in the $N \geqslant 3$ case contains terms responsible for branch cuts. These terms are suppressed by powers of $s / \Lambda$, and, naively, could have been neglected. It is true, though, that the explicit form of these terms is UV sensitive and cannot be established without the knowledge of UV physics. One should be aware of these terms since they reflect underlying physics - the presence of the infinite tower of the KK states. Fortunately, none of the results of the present work depend on these terms.

\section{THE GRAVITON PROPAGATOR}

Now it is time to turn to gravitons with their specific tensorial structure. We will consider and analyze the equation of motion of the DGP-type model presented in Eq. (8), which we reproduce here again for convenience:

$$
\begin{aligned}
& \delta^{(N)}(y) M_{\mathrm{Pl}}^{2}\left(a G_{\mu \nu}^{(4)}+b G_{\mu \nu}^{(D)}\right) \delta_{A}^{\mu} \delta_{B}^{\nu}+M_{*}^{2+N} G_{A B}^{(D)} \\
& \quad=-T_{\mu \nu}(x) \delta_{A}^{\mu} \delta_{B}^{\nu} \delta^{(N)}(y) .
\end{aligned}
$$

Here $G^{(4)}$ and $G^{(D)}$ denote the four-dimensional and $D$-dimensional Einstein tensors, respectively, while $a$ and $b$ are certain constants satisfying the constraint

$$
a+b=1 .
$$

For simplicity we choose a source term localized on the brane, namely, $T_{\mu \nu}(x) \delta^{(N)}(y)$. At the effective-theory level the ratio $a / b \equiv a /(1-a)$ is a free parameter. The only guidelines we have for its determination are (i) phenomenological viability; (ii) intrinsic self-consistency of the effective theory, which, by assumption, emerges as a low-energy limit of a self-consistent UV-completed underlying "prototheory." Specifying the prototheory would allow one to fix the ratio $a /(1-a)$ in terms of fundamental parameters.

Our task is to study the gravitational field produced by the source $T_{\mu \nu}(x) \delta^{(N)}(y)$. To this end we linearize Eq. (38). If $g_{A B} \equiv \eta_{A B}+2 h_{A B}$, in the linearized in $h$ approximation we find

$$
\begin{aligned}
G_{A B}^{(D)}= & \partial_{D}^{2} h_{A B}-\partial_{A} \partial_{C} h_{B}^{C}-\partial_{B} \partial_{C} h_{A}^{C}+\partial_{A} \partial_{B} h_{C}^{C}-\eta_{A B} \partial_{D}^{2} h_{C}^{C} \\
& +\eta_{A B} \partial_{C} \partial_{D} h^{C D},
\end{aligned}
$$

where $\partial_{D}^{2} \equiv \partial_{D} \partial^{D}$. On the other hand, the four-dimensional Einstein tensor in the linearized approximation is

$$
\begin{aligned}
G_{\mu \nu}^{(4)}= & \partial_{\beta}^{2} h_{\mu \nu}-\partial_{\mu} \partial_{\alpha} h_{\nu}^{\alpha}-\partial_{\nu} \partial_{\alpha} h_{\mu}^{\alpha}+\partial_{\mu} \partial_{\nu} h_{\alpha}^{\alpha}-\eta_{\mu \nu} \partial_{\beta}^{2} h_{\alpha}^{\alpha} \\
& +\eta_{\mu \nu} \partial_{\alpha} \partial_{\beta} h^{\alpha \beta} .
\end{aligned}
$$

In what follows we will work in the harmonic gauge,

$$
\partial^{A} h_{A B}=\frac{1}{2} \partial_{B} h_{C}^{C}
$$

The advantage of this gauge is that in this gauge the expression for $G_{A B}^{(D)}$ significantly simplifies,

$$
G_{A B}^{(D)}=\partial_{D}^{2} h_{A B}-\frac{1}{2} \eta_{A B} \partial_{D}^{2} h_{C}^{C} .
$$

Additional conditions which are invoked to solve the $\{a b\}$ and $\{a \mu\}$ components of the equations of motion are

$$
h_{a \mu}=0, \quad h_{a b}=\frac{1}{2} \eta_{a b} h_{C}^{C} .
$$

Using the last equation it is not difficult to obtain the relation

$$
N h_{\mu}^{\mu}=(2-N) h_{a}^{a} .
$$

This relation obviously suggests that we should consider separately two cases: (i) $N=2$; (ii) $N>2$. We will see, however, that the results in the $N=2$ and $N>2$ cases are somewhat similar.

\section{A. Brane-induced gravity in six dimensions $(N=2)$}

In two extra dimensions Eq. (44) implies

$$
h_{\mu}^{\mu}=0 .
$$

Therefore, the trace of the $D$-dimensional graviton coincides with the trace of the extra-dimensional part,

$$
h_{A}^{A}=h_{a}^{a} .
$$

As a result, the four-dimensional components of the harmonic gauge condition (41) reduce to

$$
\partial^{\mu} h_{\mu \nu}=\frac{1}{2} \partial_{\nu} h_{a}^{a} .
$$

Let us now have a closer look at the $\{\mu \nu\}$ part of Eq. (8). Taking the trace of this equation and using Eqs. (42), (40), (45), and (47) we arrive at ${ }^{3}$

$$
(3 b-1) \delta^{(N)}(y) M_{\mathrm{Pl}}^{2} \partial_{\mu}^{2} h_{a}^{a}+2 M_{*}^{2+N} \partial_{A}^{2} h_{a}^{a}=T_{\mu}^{\mu} \delta^{(N)}(y) .
$$

The obtained equation is very similar to the scalar-field equation (12). Therefore, we will follow the same route as in the scalar-field case, until we come to a subtle point, a would-be obstacle, which was nonexistent in the scalar-field case.

Let us Fourier-transform Eq. (48),

\footnotetext{
${ }^{3}$ As before, we put the transverse derivatives to be zero in the $\Delta$ $\rightarrow 0$ limit.
} 


$$
\begin{aligned}
& (3 b-1) \delta^{(N)}(y) M_{\mathrm{Pl}}^{2}\left(-p^{2}\right) \widetilde{h}_{a}^{a}(p, y) \\
& \quad+2 M_{*}^{2+N}\left(-p^{2}-\Delta_{y}\right) \widetilde{h}_{a}^{a}(p, y)=\widetilde{T}(p) \delta^{(N)}(y) .
\end{aligned}
$$

The general solution of the above equation is

$$
\begin{aligned}
\tilde{h}_{a}^{a}(p, y) & =\frac{\widetilde{T}(p)}{M_{\mathrm{Pl}}^{2}} \mathcal{G}(p, y), \\
\mathcal{G} & =\frac{D(p, y)}{2 m_{c}^{2}-(3 b-1) p^{2} D_{0}(p)}+c \mathcal{G}_{\mathrm{hom}},
\end{aligned}
$$

where the solution of the homogeneous equation takes the form

$$
\mathcal{G}_{\text {hom }}=D(p, y) \delta\left(2 m_{c}^{2}-(3 b-1) p^{2} D_{0}(p)\right)
$$

To begin with, let us consider the case $3 b>1$. Then the first term on the right-hand side of Eq. (51) has poles for complex values of $p^{2}$, as can be readily seen from the expressions for $D_{0}$ obtained in Sec. II. For instance, in the 6D case this pole is determined by the equation

$$
s=\frac{2 m_{c}^{2}}{(3 b-1) D_{0}(s)}=\frac{4 \pi 2 m_{c}^{2}}{(3 b-1)}\left[\ln \frac{\Lambda^{2}}{-s}\right]^{-1}
$$

This equation has at least two solutions of the form

$$
s_{*} \approx \frac{4 \pi 2 m_{c}^{2}}{3 b-1}\left[\ln \frac{\Lambda^{2}}{m_{c}^{2}}\right]^{-1}
$$

and

$$
\gamma_{1} \simeq-\frac{\pi}{\log \left(\Lambda^{2} / m_{c}^{2}\right)}, \quad \gamma_{2} \simeq 2 \pi+\frac{\pi}{\log \left(\Lambda^{2} / m_{c}^{2}\right)}
$$

The quantity $\tilde{h}_{a}^{a}(p, y)$ is not a gauge invariant variable. Therefore, the presence of certain poles in the expression for $\tilde{h}_{a}^{a}(p, y)$ depends on a gauge. However, explicit calculations (see below) show that the poles found above also enter the gauge invariant physical amplitude. Therefore, we need to take these poles seriously and analyze their physical consequences.

\section{B. $b>1 / 3$}

If $b>1 / 3$ there are no poles on the physical Riemann sheet. Instead, poles appear on the nearest nonphysical Riemann sheets. These poles cannot be identified with any physical states of the theory. They represent a signature of massive resonance states. All other complex poles appear on subsequent nonphysical Riemann sheets.

Using a contour integral one can easily write down the spectral representation for the Green's function $\mathcal{G}$

$$
\mathcal{G}(p, y=0)=\frac{1}{\pi} \int_{0}^{\infty} \frac{\rho(t) d t}{t-p^{2}-i \bar{\epsilon}},
$$

where the spectral function is defined as

$$
\rho(t)=\frac{2 m_{c}^{2} \operatorname{Im} D_{0}(t)}{\left[(3 b-1) t \operatorname{Re} D_{0}-2 m_{c}^{2}\right]^{2}+\left[(3 b-1) t \operatorname{Im} D_{0}\right]^{2}},
$$

and

$$
\operatorname{Im} D_{0}=\pi \int \frac{d^{N} q}{(2 \pi)^{N}} \delta\left(t-q^{2}\right)=\frac{\pi^{(N+2) / 2}}{(2 \pi)^{N} \Gamma(N / 2)} t^{(N-2) / 2}
$$

We see that $\rho(t)$ satisfies the positivity requirement. Equation (56) guarantees that the Green's function $\mathcal{G}$ is causal.

The next step is applying the expression for $\mathcal{G}$ to calculate $\tilde{h}_{\mu \nu}$. In fact, it is more convenient to calculate the tree-level amplitude

$$
A(p, y) \equiv \widetilde{h}_{\mu \nu}(p, y) \tilde{T}^{\prime \mu \nu}(p),
$$

where $\widetilde{T}^{\prime \mu \nu}(p)$ is a conserved energy-momentum tensor,

$$
p_{\mu} \tilde{T}^{\prime \mu \nu}=p_{\nu} \tilde{T}^{\prime \mu \nu}=0 .
$$

Using Eqs. (38), (50), and (70) we obtain the following expression for the amplitude $A(p, y)$ :

$$
\begin{aligned}
A(p, y)= & \frac{1}{M_{\mathrm{Pl}}^{2}} \frac{D(p, y)}{p^{2} D_{0}(p)-m_{c}^{2}} \\
& \times\left\{\widetilde{T}_{\mu \nu} \widetilde{T}^{\prime \mu \nu}-\frac{\widetilde{T} \widetilde{T}^{\prime}}{2}\left[\frac{(2 b-1) p^{2} D_{0}-m_{c}^{2}}{(3 b-1) p^{2} D_{0}-2 m_{c}^{2}}\right]\right\} .
\end{aligned}
$$

Let us study the above expression in some detail. The first question to ask is about poles. It is quite clear that the $p^{2}$ poles of $A$ are of two types; their position is determined by

$$
p^{2} D_{0}(p)=m_{c}^{2}
$$

or

$$
(3 b-1) p^{2} D_{0}(p)=2 m_{c}^{2} .
$$

As was explained previously, all these poles appear on the second Riemann sheet, with the additional images on other nonphysical sheets. None of these poles can be identified with asymptotic physical states. As was elucidated above, the occurrence of the poles on the second and subsequent Riemann sheets corresponds to the massive-resonance nature of the effective 4D graviton. Our previous analysis can be repeated practically verbatim, with minor modifications, proving the analyticity and causality of the amplitude $A$.

Next, we observe that at large momenta, i.e., when $p^{2} D_{0}(p) \gg m_{c}^{2}$, the scalar part of the propagator has $4 \mathrm{D}$ be- 
havior; the tensorial structure is not four dimensional, however. The terms in the braces in Eq. (60), namely,

$$
\left\{\widetilde{T}_{\mu \nu} \widetilde{T}^{\prime \mu \nu}-\frac{2 b-1}{2(3 b-1)} \tilde{T} \widetilde{T}^{\prime}\right\},
$$

correspond to the exchange of massive gravitons and scalar degrees of freedom. This would give rise to additional contributions in the light bending and is excluded phenomenologically, unless the contribution due to extra polarizations is canceled by some other interactions (such as, e.g., an additional repulsive vector exchange). Note also that when $b$ $\gg a$, i.e., $b \rightarrow 1$, one obtains the tensorial structure of $6 \mathrm{D}$ gravity, as expected from Eq. (7).

On the other hand, at large distances, i.e., at $p^{2} D_{0}(p)$ $\ll m_{c}^{2}$, we get the following tensorial structure of the amplitude (60):

$$
\left\{\widetilde{T}_{\mu \nu} \widetilde{T}^{\prime \mu \nu}-\frac{1}{4} \widetilde{T} \widetilde{T}^{\prime}\right\}
$$

This exactly corresponds to the exchange of a sixdimensional graviton, as was expected.

\section{C. $b<1 / 3$}

This case is conceptually different from that of Sec. III B. As we will see momentarily, if $b<1 / 3$ there are no problems in (i) maintaining $4 \mathrm{D}$ unitarity and (ii) getting the appropriate 4D tensorial structure of gravity at subhorizon distances. This is achieved at the price of abandoning 4D analyticity, in its standard form, which could presumably lead to the loss of causality at distances of the order of $m_{c}^{-1} \sim 10^{28} \mathrm{~cm}$. The absence of causality at distances $\gtrsim 10^{28} \mathrm{~cm}$ was argued recently [27] to be an essential ingredient for solving the cosmological constant problem.

Although all derivations and conclusions are quite similar for any ratio $a / b$ as long as $2 b<a$, we will stick to the technically simplest example $b=0, a=1$. In the situation at hand, the homogeneous part (52) need not be trivial, i.e., $c$ need not vanish. The value of the constant $c$ is determined once the rules for the pole at $p^{2} D_{0}(p)+2 m_{c}^{2}=0$ are specified. In Ref. [40] the vanishing of $c$ was postulated. This choice leads to a nonunitary Green's function. Therefore, we abandon the condition $c=0$ in an attempt to make a more consistent choice that would guarantee 4D unitarity. We stress that we are after unitarity here, not unitarity plus causality.

To begin with we pass to the Euclidean space in $p^{2}$ (i.e., $p^{2} \rightarrow p_{E}^{2}$ ) and introduce the following notation:

$$
P^{(E)}\left(p_{E}^{2}\right) \equiv \frac{1}{2 m_{c}^{2}-p_{E}^{2} D_{0}\left(p_{E}\right)-i \epsilon} .
$$

The function $P^{(E)}$ is a Euclidean-space solution of Eq. (49), with the particular choice $c=i \pi$. [The choice $c=-i \pi$ would lead to Eq. (63) with the replacement $\epsilon \rightarrow-\epsilon$.]

As the next step we will analyze the complex plane of $p_{E}^{2}$. Since the function $D_{0}\left(p_{E}\right)$ is real, the function $P^{(E)}\left(p_{E}^{2}\right)$ must (and does) have an isolated singularity in the $p_{E}^{2}$ plane which is similar to a conventional massive pole, except that it lies in the Euclidean domain. This singularity occurs at the point $p_{E}^{2}=p_{*}^{2}$ is defined by the condition

$$
p_{*}^{2} D_{0}\left(p_{*}\right)=2 m_{c}^{2}, \quad p_{*}^{2} \text { real and positive. }
$$

This is the only isolated singularity in Eq. (63); it is located in the complex $p_{E}^{2}$ plane on the real positive semiaxis. In addition to this pole singularity, the function (63) has a branch cut stretching from zero to $-\infty$ due to the imaginary part of $D_{0}\left(p_{E}^{2}\right)$ appearing at negative values of $p_{E}^{2}$. As before, this branch cut is the reflection of an infinite gapless tower of the KK states. As a result, the following spectral representation obviously emerges for $P^{(E)}\left(p_{E}^{2}\right)$ :

$$
P^{(E)}\left(p_{E}^{2}+i \epsilon\right)=\frac{1}{\pi} \int_{0}^{-\infty} \frac{\operatorname{Im} P^{(E)}(u) d u}{u-p_{E}^{2}}+\frac{R}{p_{*}^{2}-p_{E}^{2}-i \epsilon},
$$

with the Euclidean pole term being "unconventional." The residue of the pole $R$ is given (for any $N$ ) by

$$
R^{-1}=\int \frac{d^{N} q}{(2 \pi)^{N}} \frac{q^{2}}{\left(q^{2}+p_{*}^{2}\right)^{2}} .
$$

Note that in the first term on the right-hand side of Eq. (65) the integration runs from zero to minus infinity; thus, the integrand never hits the would-be pole at $u=p_{E}^{2}>0$. Therefore, the $i \epsilon$ prescription is in fact used only to specify the isolated pole at $p_{E}^{2}=p_{*}^{2}$.

We proceed further and define a symmetric function

$$
\Pi^{(E)}\left(p_{E}^{2}\right) \equiv \frac{1}{2}\left\{P^{(E)}\left(p_{E}^{2}-i \epsilon\right)+P^{(E)}\left(p_{E}^{2}+i \epsilon\right)\right\} .
$$

It is just this symmetric function on which we will focus in the remainder of the section. Let us return to Minkowski space. This is done by substituting

$$
p_{E}^{2} \rightarrow \exp (-i \pi) p^{2}, \quad u \rightarrow \exp (-i \pi) t
$$

in Eq. (65). Furthermore, observing that $\operatorname{Im} P=\operatorname{Im} \Pi$, we obtain the following representation for the Minkowskian $\Pi$ :

$$
\Pi(p)=\frac{1}{\pi} \int_{0}^{\infty} \frac{\operatorname{Im} \Pi(t) d t}{t-p^{2}-i \bar{\epsilon}}+\Pi_{0}(p),
$$

where

$$
\Pi_{0}(p) \equiv \frac{1}{2}\left(\frac{R}{p_{*}^{2}+p^{2}-i \epsilon}+\frac{R}{p_{*}^{2}+p^{2}+i \epsilon}\right) .
$$

It is necessary to emphasize that $\epsilon$ and $\bar{\epsilon}$ are two distinct regularizing parameters, $\epsilon \neq \bar{\epsilon}$. The parameter $\epsilon$ is used to regularize the pole at $p^{2}=-p_{*}^{2}$, while $\bar{\epsilon}$ sets the rules for the branch cut. The most important property of $\Pi$ is that the pole at $p^{2}=-p_{*}^{2}$ has no imaginary part, by construction. 
Hence, there is no physical particle that corresponds to this pole. In the conventional local field theory the only possible additions with no imaginary part are polynomials. Here we encounter a new structure that will be discussed in more detail at the end of this section.

Our goal is to show that a 4D-unitarity-compliant spectral representation holds for the Green's function on the brane, at least in the domain where the laws of 4D physics are applicable. To this end we turn to the function $\mathcal{G}(p, y)$, defined as

$$
\mathcal{G}=D(p, y) \Pi\left(p^{2}\right),
$$

with the purpose of studying its properties. It is convenient to pass to the momentum space with respect to extra coordinates too. Then the propagator (70) takes the form

$$
\widetilde{\mathcal{G}}(p, q)=\frac{\Pi\left(p^{2}\right)}{q^{2}-p^{2}-i \vec{\epsilon}} .
$$

With these definitions in hand, we can write down the 4D dispersion relation. We start from the Källen-Lehman representation for the propagator (71). As we will check below, this representation takes the form

$$
\widetilde{\mathcal{G}}(p, q)=\frac{1}{\pi} \int_{0}^{\infty} \frac{\operatorname{Im} \widetilde{\mathcal{G}}(t, q) d t}{t-p^{2}-i \bar{\epsilon}}+\frac{\Pi_{0}\left(p^{2}\right)-\Pi_{0}\left(q^{2}\right)}{q^{2}-p^{2}-i \bar{\epsilon}} .
$$

The imaginary part of $\widetilde{\mathcal{G}}$ is defined as follows:

$$
\operatorname{Im} \widetilde{\mathcal{G}}(t, q)=\pi \delta\left(q^{2}-t\right) \operatorname{Re} \Pi(t)+\operatorname{Im} \Pi(t) \mathcal{P} \frac{1}{q^{2}-t},
$$

where $\mathcal{P}$ stands for the principal value of a singular function,

$$
\mathcal{P} \frac{1}{q^{2}-t}=\frac{1}{2}\left(\frac{1}{q^{2}-t+i \delta}+\frac{1}{q^{2}-t-i \delta}\right) .
$$

The fact that Eq. (72) holds can be checked by substituting Eqs. (74) and (73) into Eq. (72) and exploiting the relation

$$
\begin{aligned}
\frac{1}{\pi} \int_{0}^{\infty} & \frac{\operatorname{Im} \Pi(t)}{t-p^{2}-i \bar{\epsilon}} \mathcal{P} \frac{1}{q^{2}-t} d t \\
\quad= & -\frac{\operatorname{Re} \Pi\left(q^{2}\right)-\Pi_{0}\left(q^{2}\right)+\Pi_{0}\left(p^{2}\right)-\Pi\left(p^{2}\right)}{q^{2}-p^{2}-i \bar{\epsilon}} .
\end{aligned}
$$

This turns Eq. (72) into an identity.

Finally, we approach the main point of this section-the dispersion relation for $\mathcal{G}(p, y=0)$, the Green's function on the brane. As such, it must have a spectral representation with positive spectral density, as we have already seen from the KK-based analysis. The positivity is in one-to-one correspondence with the 4D unitarity.

The dispersion relation can be obtained by integrating Eq. (72) with respect to $q$,

$$
\mathcal{G}(p, y=0)=\frac{1}{\pi} \int_{0}^{\infty} \frac{\rho(t) d t}{t-p^{2}-i \bar{\epsilon}}+\Pi_{0}\left(p^{2}\right) \operatorname{Re} D_{0}\left(-p_{*}^{2}\right)
$$

According to Eq. (72), the spectral density $\rho$ is defined as

$$
\rho(t)=\int \frac{d^{N} q}{(2 \pi)^{N}} \operatorname{Im} \widetilde{\mathcal{G}}(t, q) .
$$

The first term on the right-hand side in Eq. (76) is conventional while the second is not, and we hasten to discuss it. This term has no imaginary part, by construction. Hence, it does not contribute to the unitarity cuts in diagrams. Therefore, this term does not affect the spectral properties.

As was mentioned, in conventional $4 \mathrm{D}$ theories only a finite-order polynomial in $p^{2}$ that has no imaginary part can be added to or subtracted from the dispersion relation. This is because normally one deals with Lagrangians that contain only a finite number of derivatives, i.e., a finite number of terms with positive powers of $p^{2}$ in the momentum space. In the problem under consideration this is not the case, however. In fact, no local 4D Lagrangian exists in our model at all, and yet we are studying the spectral properties in terms of the intrinsically $4 \mathrm{D}$ variable, $p^{2}$. The theory (1) is inherently higher dimensional because of the infinite volume of the extra space. One can try to "squeeze" it in four dimensions at a price of having an infinite number of $4 \mathrm{D}$ fields. For such a theory there is no guarantee that analyticity of the Green's functions in terms of the 4D variable $p^{2}$ will hold because the effective 4D Lagrangian obtained by "integrating out" the infinite gapless KK tower will necessarily contain [5] nonlocal terms of the type $\partial^{-2}$. (Note that a similar prescription for the poles in a pure 4D local theory [45] is hard to reconcile with the path integral formulation [46]. In our case this is not a concern since the theory is not local in four dimensions in the first place.)

Therefore, it is only natural that 4D unitarity can be maintained but $4 \mathrm{D}$ analyticity cannot. Nonanalyticity leads to violation of causality, generally speaking. That is to say, the Green's function (76) is acausal. Therefore, we have an apparent violation of causality in the 4D slice of the entire (4 $+N$ )-dimensional theory which, by itself, is causal. The apparent acausal effects can manifest themselves only at the scale of the order of $m_{c}^{-1} \sim 10^{28} \mathrm{~cm}$. In fact, as was noted in [27], this is a welcome feature for a possible solution of the cosmological constant problem.

Let us now return to the first term on the right-hand side of Eqs. (76). Using Eqs. (68) and (73) we can calculate the spectral function, which comes out as follows:

$$
\rho(t)=\frac{2 m_{c}^{2} \operatorname{Im} D_{0}(t)}{\left(t \operatorname{Re} D_{0}+2 m_{c}^{2}\right)^{2}+\left(t \operatorname{Im} D_{0}\right)^{2}},
$$

where

$$
\operatorname{Im} D_{0}=\pi \int \frac{d^{N} q}{(2 \pi)^{N}} \delta\left(t-q^{2}\right)=\frac{\pi^{(N+2) / 2}}{(2 \pi)^{N} \Gamma(N / 2)} t^{(N-2) / 2} .
$$


We see that $\rho(t)$ satisfies the positivity requirement. ${ }^{4}$

Next we observe that at large momenta, i.e., at $p^{2} D_{0}(p)$ $\gg m_{c}^{2}$, the propagator we got has the desired $4 \mathrm{D}$ behavior. For the scalar part of the propagator this is expected from the studies of Sec. II. However, with regard to the tensorial structure this circumstance is less trivial. If $p^{2} D_{0}(p) \gg m_{c}^{2}$ the terms in the braces in Eq. (60),

$$
\left\{\widetilde{T}_{\mu \nu} \widetilde{T}^{\prime \mu \nu}-\frac{1}{2} \widetilde{T} \widetilde{T}^{\prime}\right\}
$$

correspond to the exchange of two physical graviton polarizations. Therefore, for the observable distances the tensorial structure of the massless $4 \mathrm{D}$ graviton (80) is recovered.

On the other hand, for large (superhorizon) distances, $p^{2} D_{0}(p) \ll m_{c}^{2}$, we get a different tensorial structure of the same amplitude,

$$
\left\{\widetilde{T}_{\mu \nu} \widetilde{T}^{\prime \mu \nu}-\frac{1}{4} \widetilde{T} \widetilde{T}^{\prime}\right\}
$$

This exactly corresponds to the exchange of the sixdimensional graviton.

\section{D. $D>6$}

Corresponding calculations and results are quite similar to those in the $D=6$ case, with minor technical distinctions which we summarize below. For $N \neq 2$

$$
h_{a b}=\frac{1}{2-N} \eta_{a b} h_{\mu}^{\mu}
$$

Therefore, we get

$$
\partial^{\mu} h_{\mu \nu}=\frac{1}{2-N} \partial_{\nu} h_{\alpha}^{\alpha}
$$

Then, the trace of the $\{\mu \nu\}$ equation takes the form

$$
k_{N} \delta^{(N)}(y) M_{\mathrm{Pl}}^{2} \partial_{\mu}^{2} h_{a}^{a}+M_{*}^{2+N}(N+2) \partial_{A}^{2} h_{a}^{a}=N T_{\nu}^{\nu} \delta^{(N)}(y),
$$

where $k_{N} \equiv 2-N(2-3 b)$. The above equation can be used to find the solution we are after. We proceed parallel to the six-dimensional case. Let us introduce the notation

$$
\widetilde{h}_{a}^{a}(p, y)=N \frac{\widetilde{T}(p)}{M_{\mathrm{Pl}}^{2}} \mathcal{G}_{N}(p, y),
$$

where

\footnotetext{
${ }^{4}$ For $N \geqslant 5$ the integral in Eq. (76) diverges. However, since our model has a manifest UV cutoff $\Lambda$, the above integral must be cut off at $\Lambda$. Alternatively, one could use a dispersion relation with subtractions.
}

$$
\mathcal{G}_{N}=\frac{D(p, y)}{-k_{N} p^{2} D_{0}(p)+u^{N}(N+2)}+c \mathcal{G}_{\mathrm{N} \mathrm{hom}} .
$$

The solution of the homogeneous equation takes the form

$$
\mathcal{G}_{\mathrm{N} \mathrm{hom}}=D(p, y) \delta\left(-k_{N} p^{2} D_{0}(p)+u^{N}(N+2)\right) .
$$

Here

$$
u^{N} \equiv M_{*}^{2+N} / M_{\mathrm{Pl}}^{2} .
$$

As in the $6 \mathrm{D}$ case, we conclude that there exists a solution to the equation

$$
-k_{N} p^{2} D_{0}(p)+u^{N}(N+2)=0
$$

with a complex value of $p^{2}$. These poles occur on the nonphysical sheets as long as $k_{N}>0$, so the Green's function admits a spectral representation.

Using the expressions above one readily calculates the tree-level amplitude $A$,

$$
\begin{aligned}
A(p, y)= & \frac{1}{M_{\mathrm{Pl}}^{2}} \frac{D(p, y)}{p^{2} D_{0}(p)-u^{N}} \\
& \times\left\{\widetilde{T}_{\mu \nu} \widetilde{T}^{\prime \mu \nu}-\frac{\widetilde{T} \widetilde{T}^{\prime}}{2}\left(\frac{\left(k_{N}-b N\right) p^{2} D_{0}-2 u^{N}}{k_{N} p^{2} D_{0}-(2+N) u^{N}}\right)\right\} .
\end{aligned}
$$

$$
\text { E. } b>(2 N-2) / 3 N
$$

In this case, there are no poles on the physical Riemann sheet. Hence, all the poles are of the resonance type. The tensorial structure at large distances is that of the $D$-dimensional theory,

$$
\left\{\widetilde{T}_{\mu \nu} \widetilde{T}^{\prime \mu \nu} \frac{1}{2+N} \widetilde{T} \widetilde{T}^{\prime}\right\} .
$$

However, the tensorial structure at short distances $\lesssim m_{c}^{-1}$ differs from that of 4D massless gravity. Hence, some additional interactions, e.g., repulsion due to a vector field, is needed to make this theory consistent with data.

$$
\text { F. } b<(2 N-2) / 3 N
$$

The consideration below is very similar to that of the $6 \mathrm{D}$ case. In perfect parallel with the $6 \mathrm{D}$ case, we consider for simplicity only the $b=0$ case and define the function

$$
P_{N}^{(E)}\left(p_{E}^{2}\right) \equiv \frac{1}{u^{N}(N+2) /(2 N-2)-p_{E}^{2} D_{0}\left(p_{E}\right)-i \epsilon},
$$

which has the spectral representation

$$
P_{N}^{(E)}\left(p_{E}^{2}+i \epsilon\right)=\frac{1}{\pi} \int_{0}^{-\infty} \frac{\operatorname{Im} P_{N}^{(E)}(u) d u}{u-p_{E}^{2}}+\frac{R}{p_{*}^{2}-p_{E}^{2}-i \epsilon} .
$$


The residue $R$ is determined by Eq. (66) while $p_{*}^{2}$ is now a solution to the equation

$$
p_{*}^{2} D_{0}\left(p_{*}\right)=\frac{u^{N}(N+2)}{2(N-1)}, \quad p_{*}^{2}>0
$$

As in the 6D situation, we use the expression (91) to define the symmetric function

$$
\Pi_{N}^{(E)}\left(p_{E}^{2}\right) \equiv \frac{1}{2}\left\{P_{N}^{(E)}\left(p_{E}^{2}-i \epsilon\right)+P_{N}^{(E)}\left(p_{E}^{2}+i \epsilon\right)\right\} .
$$

The latter, being continued to Minkowski space, admits the following spectral representation:

$$
\Pi_{N}(p)=\frac{1}{\pi} \int_{0}^{\infty} \frac{\operatorname{Im} \Pi(t) d t}{t-p^{2}-i \bar{\epsilon}}+\Pi_{0}^{(N)}(p),
$$

where

$$
\Pi_{0}^{(N)}(p) \equiv \frac{1}{2}\left(\frac{R}{p_{*}^{2}+p^{2}-i \epsilon}+\frac{R}{p_{*}^{2}+p^{2}+i \epsilon}\right)
$$

As previously, $\epsilon$ and $\bar{\epsilon}$ are two distinct regularizing parameters, $\epsilon \neq \bar{\epsilon}$.

For the Green's function of interest

$$
\mathcal{G}_{N}=\frac{D(p, y) \Pi_{N}\left(p^{2}\right)}{2 N-2},
$$

we repeat the analysis of Secs. III A, III B, and III C to confirm with certainty that the function $\mathcal{G}_{N}(p, y=0)$ does admit the spectral representation (76), with a positive spectral function, similar to the $6 \mathrm{D}$ case [see Eq. (78)].

The expression in Eq. (88) interpolates between the fourdimensional and $D$-dimensional patterns. This was already established for the scalar part of the amplitude in Sec. II. Let us examine the tensorial part. For $p^{2} D_{0}(p) \gg u^{N}$ we get

$$
\left\{\widetilde{T}_{\mu \nu} \widetilde{T}^{\mu \nu}-\frac{1}{2} \widetilde{T} \widetilde{T}^{\prime}\right\}
$$

This corresponds to two helicities of the 4D massless graviton. In the opposite limit, $p^{2} D_{0}(p) \ll u^{N}$, we recover the tensorial structure corresponding to the $(4+N)$-dimensional massless graviton.

\section{PERTURBATION THEORY IN MASSIVE GRAVITY: HARD MASS VS SOFT}

We start from a brief review of a well-known phenomenon - the breakdown of perturbation theory for a graviton with hard mass [34], occurring at a scale lower than the UV cutoff of the theory [35-37]. We then elucidate as to how this problem is avoided in the models (1),(7).

The 4D action of a massive graviton is

$$
S_{m}=\frac{M_{\mathrm{Pl}}^{2}}{2} \int d^{4} x \sqrt{g} R(g)+\frac{M_{\mathrm{Pl}}^{2} m_{g}^{2}}{2} \int d^{4} x\left[h_{\mu \nu}^{2}-\left(h_{\mu}^{\mu}\right)^{2}\right],
$$

where $m_{g}$ stands for the graviton mass and $h_{\mu \nu} \equiv\left(g_{\mu \nu}\right.$ $\left.-\eta_{\mu \nu}\right) / 2$. The mass term has the Pauli-Fierz form [34]. This is the only Lorentz invariant form of the mass term which at quadratic order in $h_{\mu \nu}$ does not give rise to ghosts [47]. Higher powers in $h$ could be arbitrarily added to the mass term since there is no principle, such as reparametrization invariance, which could fix these terms. Hence, for definiteness, we assume that the indices in the mass term are raised and lowered by $\eta_{\mu \nu}$. Had we used $g_{\mu \nu}$ instead, the difference would appear only in cubic and higher orders in $h$, which are not fixed anyway.

In order to reveal the origin of the problem, let us have a closer look at the free graviton propagators in the massless and massive theory. For the massless graviton we find

$$
D_{\mu \nu ; \alpha \beta}^{0}(p)=\left(\frac{1}{2} \bar{\eta}_{\mu \alpha} \bar{\eta}_{\nu \beta}+\frac{1}{2} \bar{\eta}_{\mu \beta} \bar{\eta}_{\nu \alpha}-\frac{1}{2} \bar{\eta}_{\mu \nu} \bar{\eta}_{\alpha \beta}\right) \frac{1}{-p^{2}-i \epsilon},
$$

where

$$
\bar{\eta}_{\mu \nu} \equiv \eta_{\mu \nu}-\frac{p_{\mu} p_{\nu}}{p^{2}}
$$

The momentum-dependent parts of the tensor structure were chosen in a particular gauge convenient for our discussion. On the other hand, there is no gauge freedom for the massive gravity presented by the action (98); hence the corresponding propagator is unambiguously determined,

$$
\begin{aligned}
D_{\mu \nu ; \alpha \beta}^{m}(p)= & \left(\frac{1}{2} \tilde{\eta}_{\mu \alpha} \tilde{\eta}_{\nu \beta}+\frac{1}{2} \tilde{\eta}_{\mu \beta} \tilde{\eta}_{\nu \alpha}-\frac{1}{3} \tilde{\eta}_{\mu \nu} \tilde{\eta}_{\alpha \beta}\right) \\
& \times \frac{1}{m_{g}^{2}-p^{2}-i \epsilon},
\end{aligned}
$$

where

$$
\tilde{\eta}_{\mu \nu} \equiv \eta_{\mu \nu}-\frac{p_{\mu} p_{\nu}}{m_{g}^{2}}
$$

We draw the reader's attention to the $1 / m_{g}^{4}, 1 / m_{g}^{2}$ singularities of the above propagator. The fact of their occurrence will be important in what follows.

It is the difference in the numerical coefficients in front of the $\eta_{\mu \nu} \eta_{\alpha \beta}$ structure in the massless vs massive propagators $(1 / 2$ versus $1 / 3)$ that leads to the famous perturbative discontinuity [48-50]. No matter how small the graviton mass is, the predictions are substantially different in the two cases. The structure (101) gives rise to contradictions with observations.

However, as was first pointed out in Ref. [35], this discontinuity could be an artifact of relying on the tree-level 
perturbation theory which, in fact, badly breaks down at a higher nonlinear level $[35,36]$. One should note that the discontinuity does not appear on curved backgrounds [51,52] another indication of the spurious nature of the "mass discontinuity phenomenon."

To see the failure of the perturbative expansion in the Newton constant $G_{N}$, one could examine the Schwarzschild solution of the model (98), as was done in Ref. [35] (see also [53-55]). However, probably the easiest way to understand the perturbation theory breakdown is through examination of the tree-level trilinear graviton vertex diagram. At the nonlinear level we have two extra propagators which could provide a singularity in $m_{g}$ up to $1 / \mathrm{m}_{g}^{8}$.

Two leading terms $1 / m_{g}^{8}$ and $1 / m_{g}^{6}$ do not contribute [36], so that the worst singularity is $1 / \mathrm{m}_{g}^{4^{g}}$. This is enough to lead to the perturbation theory breakdown. For a Schwarzschild source of mass $M$ the breakdown happens $[35,36]$ at the scale

$$
\Lambda_{m} \sim m_{g}\left(M m_{g} / M_{\mathrm{Pl}}^{2}\right)^{-1 / 5} .
$$

The result can also be understood in terms of interactions of longitudinal polarizations of the massive graviton, which become strong [37]. For the gravitational sector per se, the corresponding scale $\Lambda_{m}$ reduces to [37]

$$
m_{g}\left(m_{g} / M_{\mathrm{Pl}}\right)^{-1 / 5} \text {. }
$$

If one uses the freedom associated with possible addition of higher nonlinear terms, one can make [37] the breaking scale as large as

$$
m_{g} /\left(m_{g} / M_{\mathrm{Pl}}\right)^{1 / 3} .
$$

(Note that at the classical level the strong-coupling problem of PF gravity can be evaded by summing up tree-level nonlinear diagrams $[35,36]$. To determine whether the problem is present at the quantum level, one must perform perturbations on a stable background; however, the Minkowski-space background is not stable for PF gravity, with the instability setting in almost instantaneously [39]. For recent discussions of massive gravity see Refs. [56,57].)

Summarizing, in the diagrammatic language the reason for the precocious breakdown of perturbation theory can be traced back to the infrared terms in the propagator (101), which scale as

$$
\frac{p_{\mu} p_{\nu}}{m_{g}^{2}}
$$

These terms do not manifest themselves at the linear level; however, they do contribute to nonlinear vertices creating problems in the perturbative treatment of massive gravity already in a classical theory.

We will see momentarily that similar problems are totally absent in the propagator of the model (1). For illustrational purposes it is sufficient to treat the $N=2$ case. All necessary calculations were carried out in Sec. III. Therefore, here we just assemble relevant answers.

For $N=2$ and $b>1 / 3$ we find

$$
\frac{p_{\mu} p_{\nu} D(p, y)}{2 m_{c}^{2}-(3 b-1) p^{2} D_{0}(p)+i \epsilon} .
$$

In the limit $m_{c} \rightarrow 0$ the above expression, as opposed to Eq. (103), is regular. Similar calculations can be done in the $N$ $>2$ case. The result is proportional to

$$
\frac{p_{\mu} p_{\nu} D(p, y)}{(2+N) u^{N}-k_{N} p^{2} D_{0}(p)+i \epsilon},
$$

which is also regular in the $m_{c} \rightarrow 0$ limit where it approaches the $4 \mathrm{D}$ expression. Therefore, we conclude that there is no reason to expect any breaking of perturbation theory in the model (1) below the scale of its UV cutoff.

If $b<1 / 3$ and $N=2$ we find, by the same token,

$$
\frac{p_{\mu} p_{\nu} D(p, y)}{2}\left(\frac{1}{2 m_{c}^{2}-(3 b-1) p^{2} D_{0}(p)+i \epsilon}+(\epsilon \rightarrow-\epsilon)\right) \text {. }
$$

Again, in the limit $m_{c} \rightarrow 0$, the above expression, in contradistinction with Eq. (103), is regular. Moreover, in this limit (and at $y=0$ ) it approaches the $4 \mathrm{D}$ expression, in a particular gauge. Analogous calculations can be readily done in the $N$ $>2$ and $b<(2 N-2) / 3 N$ case. The result is

$$
\frac{p_{\mu} p_{\nu} D(p, y)}{2}\left[\frac{1}{(2+N) u^{N}-k_{N} p^{2} D_{0}(p)+i \epsilon}+(\epsilon \rightarrow-\epsilon)\right] .
$$

This expression is also regular in the $m_{c} \rightarrow 0$ limit where it arrives at the correct 4D limit. We conclude, therefore, that in the general case there is no reason to expect any breaking of perturbation theory in the model (7) below the scale of its UV cutoff. Note that the expressions (106) and (107) are singular for small Euclidean momenta $p^{2} \sim-m_{c}^{2}$. By construction this singularity has no imaginary part and there is no physical state associated with it. One might expect that this singularity will be removed after the loop corrections are taken into account in a full quantum theory. These considerations are beyond the scope of the present work.

An analogy with the Higgs mechanism for non-Abelian gauge fields is in order here. For massive non-Abelian gauge fields nonlinear amplitudes violate the unitarity bounds at the scale set by the gauge field mass. This disaster is cured through the introduction of the Higgs field. Likewise, nonlinear amplitudes of the 4D massive gravity (98) blow up at the scale $\Lambda_{m}$. The unwanted explosion is canceled at the expense of introducing an infinite number of the KaluzaKlein fields in Eq. (1).

\section{DISCUSSION AND CONCLUSIONS}

In the present work we studied the model (7) of braneinduced gravity in codimensions 2 and higher. This model has stringent and testable predictions. Gravity is modified at short distances, of the order of $\sim 0.1 \mathrm{~mm}$ or so, and simul- 
taneously at ultralarge distances, of the order of $\sim 10^{29} \mathrm{~mm}$, give or take an order of magnitude. The short-distance modification can be tested in tabletop gravitational experiments $[58,59]$. Modification of gravity at a millimeter scale and its relation to the cosmological constant problem was first discussed in Ref. [60].

The modification of gravity at a millimeter scale in the present model (1) is a consequence of the large-distance modification and vice versa. These are two faces of one and the same phenomenon. However, we should point out that technically and conceptually the approaches to the cosmological constant problem discussed in Ref. [60], on the one hand, and Refs. [2,5], on the other, are rather different- the former relies on the short-distance (UV) modification of gravity, while the latter is entirely based on the large-distance (IR) modification of gravity.

The large-distance modification of gravity can manifest itself in cosmological solutions. The case $b=0$ and $a=1$ seems to be most interesting for these purposes. As we argued in the present work, it leads to apparent violations of 4D causality which could manifest themselves at the scales of the order of today's Hubble scale. Manifestations of acausality might be tested in cosmological observations. In particular, such an acausal theory might be the reason behind the smallness of the observable space-time curvature [27].

It is instructive to point out how the $b=0, a=1$ model evades a well-known no-go theorem for massive gravity [49]. Let us first briefly recall the theorem. A 4D massive graviton has extra polarizations, one of which couples to sources in the leading order in a weak field. The additional attraction due to this polarization is observationally unacceptable and has to be canceled. This can be achieved by introducing a ghost that gives rise to a compensating repulsive force [49]. Hence, one ends up either with a theory that has a ghost or with a theory that has no ghosts but is phenomenologically unacceptable. This is the essence of the no-go theorem [49]. The theorem can easily be generalized for a theory with an infinite number of states [61]. In the latter case, in order to obtain a phenomenologically acceptable theory of a massive graviton at observable distances, one should give up positivity of the spectral function in the dispersion relation for the corresponding Green's function. This would violate unitarity of the model. However, the above argument assumes 4D analyticity, the consequence of which is the existence of a spectral representation for the Green's function. In our case 4D analyticity is violated, and so are the conditions of the no-go theorem.

We would like to point out a certain common feature with $(2+1)$ topologically massive gauge and gravity theory [62] where the large-distance interactions are also powerlike.

Finally, we would like to emphasize that the models (1) and (7) give rise to a gravitational analogue of the Higgs mechanism in the following sense- the effective gravitonmediating interaction is massive, and, nevertheless, the growth of the nonlinear amplitudes is softened at the expense of having an infinite number of fields. This phenomenon can be rather transparently understood from the standpoint of the $\mathrm{KK}$ modes. The manifest reparametrization invariance is a convenient bookkeeping tool in this case for determining whether or not the amplitudes blow up. The reparametrization invariance at each $\mathrm{KK}$ level is maintained at the same KK level only in the linearized approximation. Nonlinear effects mix distinct KK levels under the reparametrization transformations $[63,64]$. Hence, if the KK tower is truncated at some finite level, the breakdown of perturbation theory in nonlinear diagrams is inevitable. However, if the infinite totality of the KK modes are kept, as in Eq. (1), the softening of the amplitudes should be expected. The present work fully confirms this expectation. In light of this finding, it would be interesting to discuss the strong-coupling issue in nonlinear interactions of the 5D DGP model studied in Refs. $[36,65,66]$. In particular, the immediate task is to understand whether this is a problem of the particular perturbation theory, as advocated in Ref. [36], or a problem inherent in the model itself $[65,66]$. Already, from the equations of the present work, it is clear that the $N=1$ DGP model with $b$ $=1$ in Eq. (7) has no strong-coupling problem. The other possibilities are currently under investigation; the answers will be reported elsewhere.

\section{ACKNOWLEDGMENTS}

We would like to thank Ignatios Antoniadis, Leonardo Giusti, Gia Dvali, Massimo Porrati, Arkady Vainshtein, Pierre Vanhove, and Gabriele Veneziano for useful discussions. A significant part of this work was carried out while both authors were at CERN. We are grateful to the CERN Theory Division for kind hospitality. The work of M.S. was supported in part by DOE Grant No. DE-FG02-94ER408.
[1] G. Dvali, G. Gabadadze, and M. Porrati, Phys. Lett. B 485, 208 (2000).

[2] G.R. Dvali and G. Gabadadze, Phys. Rev. D 63, 065007 (2001).

[3] M. Kolanovic, M. Porrati, and J.W. Rombouts, Phys. Rev. D 68, 064018 (2003).

[4] I. Antoniadis, R. Minasian, and P. Vanhove, Nucl. Phys. B648, 69 (2003)

[5] G. Dvali, G. Gabadadze, and M. Shifman, Phys. Rev. D 67, 044020 (2003); in Continuous Advances in QCD 2002, edited by K. Olive, M. Shifman, and M. Voloshin (World Scientific, Singapore, 2002), pp. 566-581.
[6] O. Corradini, A. Iglesias, and Z. Kakushadze, Int. J. Mod. Phys. A 18, 3221 (2003); A. Iglesias and Z. Kakushadze, "A Novel Approach to the Cosmological Constant Problem," hep-th/0306297.

[7] C. Deffayet, Phys. Lett. B 502, 199 (2001).

[8] C. Deffayet, G.R. Dvali, and G. Gabadadze, Phys. Rev. D 65, 044023 (2002).

[9] R. Dick, Class. Quantum Grav. 18, R1 (2001); Acta Phys. Pol. B 32, 3669 (2001).

[10] R. Cordero and A. Vilenkin, Phys. Rev. D 65, 083519 (2002).

[11] A. Lue, Phys. Rev. D 66, 043509 (2002).

[12] A. Gruzinov, "On the Graviton Mass," astro-ph/0112246. 
[13] C. Deffayet, S.J. Landau, J. Raux, M. Zaldarriaga, and P. Astier, Phys. Rev. D 66, 024019 (2002).

[14] C. Deffayet, G.R. Dvali, G. Gabadadze, and A. Lue, Phys. Rev. D 64, 104002 (2001).

[15] A. Lue, Phys. Rev. D 67, 064004 (2003).

[16] A. Lue and G. Starkman, Phys. Rev. D 67, 064002 (2003); G. Dvali, A. Gruzinov, and M. Zaldarriaga, ibid. 68, 024012 (2003).

[17] G.R. Dvali, G. Gabadadze, and M.A. Shifman, Phys. Lett. B 497, 271 (2001).

[18] G. Kofinas, J. High Energy Phys. 08, 034 (2001); G. Kofinas, E. Papantonopoulos, and I. Pappa, Phys. Rev. D 66, 104014 (2002).

[19] G. Kofinas, E. Papantonopoulos, and V. Zamarias, Phys. Rev. D 66, 104028 (2002); Astrophys. Space Sci. 283, 685 (2003).

[20] K. Maeda, S. Mizuno, and T. Torii, Phys. Rev. D 68, 024033 (2003).

[21] M. Kolanovic, Phys. Rev. D 67, 106002 (2003); 65, 124005 (2002).

[22] T. Tanaka, Phys. Rev. D 69, 024001 (2004).

[23] C. Middleton and G. Siopsis, "The Schwarzschild Solution in the DGP Model," hep-th/0311070.

[24] A. Lue, R. Scoccimarro, and G. Starkman, Phys. Rev. D 69, 044005 (2004).

[25] G.R. Dvali, G. Gabadadze, M. Kolanovic, and F. Nitti, Phys. Rev. D 64, 084004 (2001); 65, 024031 (2002).

[26] G. Dvali, G. Gabadadze, X. Hou, and E. Sefusatti, Phys. Rev. D 67, 044019 (2003).

[27] N. Arkani-Hamed, S. Dimopoulos, G. Dvali, and G. Gabadadze, "Non-Local Modification of Gravity and the Cosmological Constant Problem," hep-th/0209227.

[28] S.M. Carroll, V. Duvvuri, M. Trodden, and M.S. Turner, "Is Cosmic Speed-Up due to New Gravitational Physics?" astro-ph/0306438.

[29] T.R. Taylor and G. Veneziano, Phys. Lett. B 228, 311 (1989); Nucl. Phys. B345, 210 (1990).

[30] I. Antoniadis and E. Mottola, Phys. Rev. D 45, 2013 (1992); I. Antoniadis, P.O. Mazur, and E. Mottola, Nucl. Phys. B388, 627 (1992).

[31] M. Carena, A. Delgado, J. Lykken, S. Pokorski, M. Quiros, and C.E. Wagner, Nucl. Phys. B609, 499 (2001).

[32] E. Kiritsis, N. Tetradis, and T.N. Tomaras, J. High Energy Phys. 08, 012 (2001).

[33] I. Antoniadis, G. Gabadadze, and P. Vanhove (in progress).

[34] M. Fierz, Helv. Phys. Acta 12, 3 (1939); M. Fierz and W. Pauli, Proc. R. Soc. London 173A, 211 (1939).

[35] A.I. Vainshtein, Phys. Lett. 39B, 393 (1972).

[36] C. Deffayet, G.R. Dvali, G. Gabadadze, and A.I. Vainshtein, Phys. Rev. D 65, 044026 (2002).

[37] N. Arkani-Hamed, H. Georgi, and M.D. Schwartz, Ann. Phys. (N.Y.) 305, 96 (2003).

[38] D.G. Boulware and S. Deser, Phys. Rev. D 6, 3368 (1972).
[39] G. Gabadadze and A. Gruzinov, "Graviton Mass or Cosmological Constant?" hep-th/0312074.

[40] S.L. Dubovsky and V.A. Rubakov, Phys. Rev. D 67, 104014 (2003).

[41] R.J. Eden, P.V. Lanshoff, D.I. Olive, and J.C. Polkinghorne, The Analytic S-Matrix (Cambridge University Press, Cambridge, England, 1966), p. 247.

[42] R. Gregory, V.A. Rubakov, and S.M. Sibiryakov, Phys. Rev. Lett. 84, 5928 (2000).

[43] C. Csaki, J. Erlich, and T.J. Hollowood, Phys. Rev. Lett. 84, 5932 (2000); C. Csaki, J. Erlich, T.J. Hollowood, and J. Terning, Phys. Rev. D 63, 065019 (2001).

[44] G.R. Dvali, G. Gabadadze, and M. Porrati, Phys. Lett. B 484, 112 (2000).

[45] T.D. Lee and G.C. Wick, Nucl. Phys. B9, 209 (1969).

[46] D.G. Boulware and D.J. Gross, Nucl. Phys. B233, 1 (1984).

[47] P. Van Nieuwenhuizen, Nucl. Phys. B60, 478 (1973).

[48] Y. Iwasaki, Phys. Rev. D 2, 2255 (1970).

[49] H. van Dam and M.J. Veltman, Nucl. Phys. B22, 397 (1970).

[50] V.I. Zakharov, JETP Lett. 12, 312 (1970).

[51] I.I. Kogan, S. Mouslopoulos, and A. Papazoglou, Phys. Lett. B 503, 173 (2001).

[52] M. Porrati, Phys. Lett. B 498, 92 (2001).

[53] C. Aragone and J. Chela Flores, Nuovo Cimento Soc. Ital. Fis., A 10A, 818 (1972); J. Chela Flores, Int. J. Theor. Phys. 10, 103 (1974).

[54] A. Salam and J. Strathdee, Phys. Rev. D 16, 2668 (1977).

[55] T. Damour, I.I. Kogan, and A. Papazoglou, Phys. Rev. D 67, 064009 (2003).

[56] C. Deffayet and J. Mourad, Phys. Lett. B 589, 48 (2004); Class. Quantum Grav. 21, 1833 (2004).

[57] Z. Chacko, M. Graesser, C. Grojean, and L. Pilo, "Massive Gravity on a Brane," hep-th/0312117.

[58] EOT-WASH Group Collaboration, E.G. Adelberger, "SubMillimeter Tests of the gravitational Inverse Square Law," hep-ex/0202008; J.C. Long and J.C. Price, C. R. Phys. 4, 337 (2003).

[59] S. Dimopoulos and A.A. Geraci, Phys. Rev. D 68, 124021 (2003).

[60] R. Sundrum, J. High Energy Phys. 07, 001 (1999); Phys. Rev. D 69, 044014 (2004).

[61] G.R. Dvali, G. Gabadadze, and M. Porrati, Phys. Lett. B 484, 129 (2000).

[62] S. Deser, R. Jackiw, and S. Templeton, Ann. Phys. (N.Y.) 140, 372 (1982); 185, 406(E) (1988); 281, 409 (1988); 281, 409 (2000).

[63] C.R. Nappi and L. Witten, Phys. Rev. D 40, 1095 (1989).

[64] M.J. Duff, C.N. Pope, and K.S. Stelle, Phys. Lett. B 223, 386 (1989).

[65] M.A. Luty, M. Porrati, and R. Rattazzi, J. High Energy Phys. 09, 029 (2003).

[66] V.A. Rubakov, "Strong Coupling in Brane-Induced Gravity in Five Dimensions," hep-th/0303125. 